
\title{
is Research Square \\ Validity as a Measure of Data Quality in Internet of Things Systems
}

Rishabh Deo Pandey ( $\square$ phdcs10056.19@bitmesra.ac.in )

Birla Institute of Technology

Itu Snigdh

Birla Institute of Technology

\section{Research Article}

Keywords: Internet of Things (IoT), Validity, Data Freshness, Completeness, Availability, Usability.

Posted Date: November 19th, 2021

DOI: https://doi.org/10.21203/rs.3.rs-811543/v1

License: (a) This work is licensed under a Creative Commons Attribution 4.0 International License. Read Full License 


\title{
Validity as a measure of Data Quality in Internet of Things Systems
}

\author{
Rishabh Deo Pandey \\ Dept of CSE, BIT Mesra \\ phdcs10056.19@bitmesra.ac.in \\ Itu Snigdh \\ Dept. of CSE, BIT Mesra \\ itusnigdh@bitmesra.ac.in
}

\begin{abstract}
Data quality became significant with the emergence of data warehouse systems. While accuracy is intrinsic data quality, validity of data presents a wider perspective, which is more representational and contextual in nature. Through our article we present a different perspective in data collection and collation. We focus on faults experienced in data sets and present validity as a function of allied parameters such as completeness, usability, availability and timeliness for determining the data quality. We also analyze the applicability of these metrics and apply modifications to make it conform to IoT applications. Another major focus of this article is to verify these metrics on aggregated data set instead of separate data values. This work focuses on using the different validation parameters for determining the quality of data generated in a pervasive environment. Analysis approach presented is simple and can be employed to test the validity of collected data, isolate faults in the data set and also measure the suitability of data before applying algorithms for analysis.
\end{abstract}

\section{Keywords}

\section{Internet of Things (IoT), Validity, Data Freshness, Completeness, Availability, Usability.}

\section{Introduction}

Internet of Things (IoT) is a network of physical devices or things embedded with electronic devices such as sensors, actuators, software and an interconnecting network which enables it to collect and manage data without involving human interaction [1]. Common to all the mentioned applications, the IoT application comprises of four major segments [2]. The sensor and actuators with the communication capabilities are the integral part and mostly hardware oriented. The software segment consists of the user interface modules, connectivity and adaptability of the apps across different machine capabilities and interfaces [3]. IoT combines various technologies into a single intelligent unit. This intelligence in IoT networks is attributed to the amount and quality of data. All IoT devices generate varied amounts of data at varied levels of scalability and time dependence like a point of sales terminal, distributed sensors or industrial machinery [4]. These data range from being mission critical to custom business logic. Also, with the proliferation of organizations in to the IoT domains and its enablement through hardware, software services and connectivity, the complexity of the applications have increased. In addition 
to handling increased software complexity, IoT performance also depends on its responsiveness and real time digital services. Since an important part of an IoT application is the software in addition to its hardware, evaluation of the performance of an IoT application becomes necessary [5]. Software metrics therefore need to be developed for IoT applications, in order to monitor IoT performance for fulfilling the following requirements:

- Able to monitor devices that run on different processor architecture.

- Able to monitor IoT application written in different programming languages.

- Overhead in monitoring should be minimal.

- Able to receive data generated

It is recognized that Software metrics is a measure of characteristics of the software that are quantifiable or countable. Also, the main objective of any software metric is to analyze the product or process; to determine its quality and suggest improvements as well as predict when the software development process is over [6].

In this paper, we try to determine data quality in an IoT application on the basis of different metrics namely, validity, data freshness, completeness, usability and availability. These metrics have been studied using two separate data sets. The major focus of this paper is to verify these metrics on aggregated data set instead of separate data values. In order to determine performance of a software model, we have predefined ISO-9126 Software Quality Characteristics which is used for evaluating the software model [7].

\subsection{Role of data and its quality in IoT applications}

Analysis of IoT data can be helpful in many ways such as optimizing operations, efficient use of energy and resources. Combining IoT with data analytics becomes effective in real time applications like healthcare, telematics, smart cities and the like.[8]. We have a large number of real time applications available in the market which are fully dependent on the data for its functioning. Therefore, if the data quality degrades it will have a negative impact on the customer relationship. To implement any IoT based application, large number of sensors are deployed which further generate huge amount of data. This data generally falls under the category of big data since it justifies the properties of big data namely volume, velocity and variety. Data obtained from the sensors needs to be validated before using it for any decisionmaking process [9]. Also, IoT application domains like utilities, mobile and enterprises require continuous monitoring of the data as any type of fault can lead to wrong analysis, thereby leading to decrease in trust by the customer. Apart from affecting the reasoning capability, it also leads to wastage of energy since in case of wireless sensor nodes, every bit of transmission consumes energy. [10] In addition, IoT based monitoring applications require alerting capabilities that are sophisticated and customizable considering the most decentralized network 
of devices or the inherent excessive dynamics of the network and devices. The volume of telemetry data that is collected from IoT devices are impacted by the intermittent connectivity issues [11]. Therefore, the traditional alerting tools face challenges which can be overcome to an extent by measuring the quality of the data collected. We are also aware that every IoT application is unique with a said functionality and rarely uses off-the-shelf products [12]. Therefore, a generalized solution "one-size-fits-all" is not applicable.

\section{Methodology for analysis of Metrics}

We consider "Temperature" as a common attribute that forms a part of most applications varying from personalized healthcare, to environment monitoring. The data quality dimensions related to parameter "temperature" can be illustrated as figure 1.

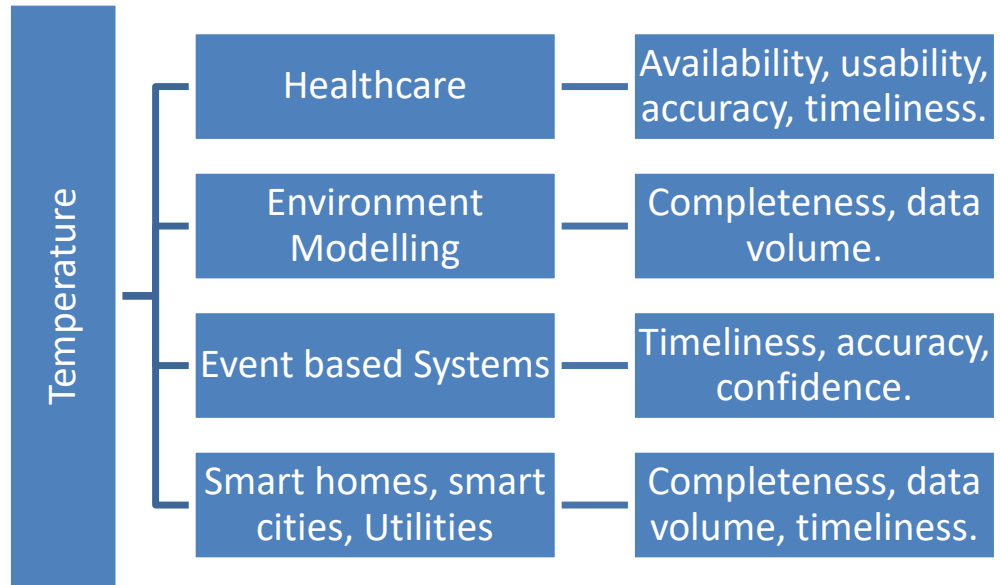

Figure 1: Data Quality requirements for different Applications.

Whenever we apply the concept of IoT for studying environmental behavior in any geographical location, large number of sensors are deployed. The data obtained from these sensor nodes go through various issues such as hardware \& software problem, poor connectivity, environmental effects [13]. Thus, the data samples obtained needs to be validated. Several types of faults occur which needs to be sorted for data validation. In this paper, we focus on two classes of faults; outof-range faults and struck-at faults.

1. Out-of-range faults occur when the data obtained from the sensors doesn't lie within an expected range of values. Generally, in this type of fault we compare using a threshold limit which is usually based on domain knowledge.

Input: Actual and Obtained maximum temperature for different months in a year.

Output: Number of invalid entries that suffer out of range faults.

count $_{\text {invalid }}=0$ 
$\mathrm{n}=$ number of months;

for $\mathrm{i} \leftarrow 0$ to $\mathrm{n}$ do $\mathrm{i} \leftarrow \mathrm{i}+1$

difference $[\mathrm{i}]=\mid$ actual $[\mathrm{i}]-$ obtained $[\mathrm{i}] \mid$

end for

// Average and SD is obtained for difference data set and limits are defined.

$\delta_{\text {lower }}=$ Average $-\mathrm{SD} ; / /$ lower threshold value

$\delta_{\text {upper }}=$ Average $+\mathrm{SD} ; / /$ upper threshold value

for $\mathrm{i} \leftarrow 0$ to $\mathrm{n}$ do $\mathrm{i} \leftarrow \mathrm{i}+1$

if $\left(\right.$ difference $[\mathrm{i}]<\delta_{\text {lower }}$ or difference[i] $\left.>\delta_{\text {upper }}\right)$

then count $_{\text {invalid }} \leftarrow$ count $_{\text {invalid }}+1$

end if

end for

end

2. Struck-at faults or constant faults occur when the data obtained from the sensor nodes show no change or very little change for a significant amount of time. It behaves as if data has reached in a frozen state and shows no variation.

Input: Maximum temperature readings of a month.

Output: Number of updates which acts as a parameter to determine struck-at faults or constant faults.

count $_{\text {update }}=0$;

$\mathrm{n}=$ number of days in a month;

// Average and SD is obtained for a month data set.

$\delta_{\text {lower }}=$ Average $-\mathrm{SD} ; / /$ lower threshold value

$\delta_{\text {upper }}=$ Average $+\mathrm{SD} ; / /$ upper threshold value

for $\mathrm{i} \leftarrow 0$ to $\mathrm{n}$ do $\mathrm{i} \leftarrow \mathrm{i}+1$

if (temperature[i] $<\delta_{\text {lower }}$ or temperature $[\mathrm{i}]>\delta_{\text {upper }}$ ) 
then count $_{\text {update }} \leftarrow$ count $_{\text {update }}+1 / /$ more the value of count $t_{\text {update }}$ less will be the chances of constant fault.

end if

end for

end

\section{Metrics used}

\subsection{Validity:}

When we are dealing with data related to pervasive environment validity is defined on the basis of some practical considerations. To determine validity of any measure, various techniques are used. For example, a database application comprising of student details, can be validated by cross-checking from various other available data sources. However, when we consider the case of IoT applications, it is not easy to obtain correctness of data since these data are generated for a short span of time. For defining validity first of all we define validity rules. Validity rule (V) can be expressed as a Boolean function. If validity rule $\mathrm{V}$ is satisfied by the data object then its value is 1 else 0 . These rules are domain specific [14-19]. The two most common validity rules are:

a. Static rules that are satisfied by verifying the data set. For example temperature in Polar region will be negative or around zero degree Celsius.

b. Dynamic rules are used to verify the changes in the data i.e. drop of 10 degrees isn't possible in half an hour.

Validity can also be expressed as a stochastic measure when it is evaluated for a particular observation period. Let $n$ be the number of updated that have occurred and $n^{\text {valid }}$ be the total valid instances; probability of validity can be expressed as:

$$
\text { Prob. Validity }=\frac{n^{\text {valid }}}{n}
$$

In research application areas, validity refers to how accurately a particular method measures any quantity. So, when we are dealing with data related to pervasive environment, validity is defined on the basis of some practical considerations that we try to explore in our work. The requirements of IoT applications are varied and the data quality dimensions align accordingly. For, example, a timely data may not be accurate while an accurate data may not be complete or in time. These tradeoffs force us to outline a metric system that could map single attribute "validity" in terms of its timeliness, completeness and its usability [20].

We assume IoT monitoring applications which employ several sensors that collect temperature data for storage and analysis. For the ease of elaboration, our focus of study considers 
'Maximum' temperature. Since we consider data for an entire year, we also focus on evaluating metrics for aggregated data in our study. We usually obtain large set of temperature data because it keeps on fluctuating. Therefore, large volume of data will be obtained for a year. The data sets $[21,22]$ which has been used for evaluating validity is obtained from the meteorological lab, in the district of Ranchi, India [23.3441 $\left.\mathrm{N}, 85.3096^{\circ} \mathrm{E}\right]$. It consists of maximum and minimum temperature of all months for the year 2012. We have considered maximum temperature as a measure for determining its validity. For deciding the validity of a dataset, first of all we need to calculate the update_value which serves as an important entity for validation of any data. To obtain update_value, we followed following steps:

- Step 1: Obtain mean of maximum temperature for each month and calculate Standard Deviation (SD) for the dataset.

- Step 2: Set the limits with the help of obtained mean and SD values.

- Step 3: To determine updates in a month, check those temperature values which doesn't lie in the limit.

- Step 4: Calculate the different update_value for each month which is the difference between the consecutive updates in terms of the duration.

On the basis of these values, max update_value for each month was determined which is depicted in figure 2. From the figure we see that there are two discrepancies in the graph, in the month of February and July which should ideally be aligned as they belong to the same period and same geographical location. Thus, any irrelevance is an anomaly.

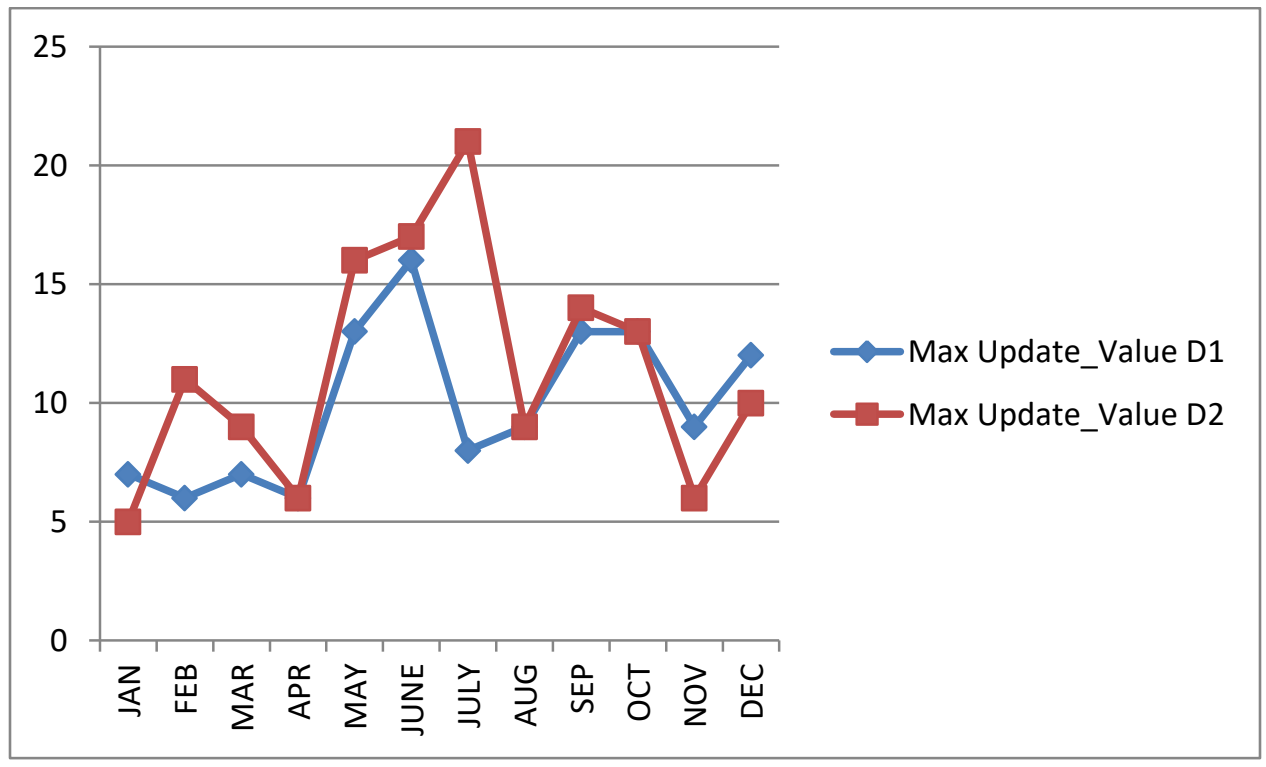

Figure 2: shows maximum update value determined for each month.

To determine validity, we used weather data [23] and our algorithm compared the max temperature value. Then, we isolated the particular day in a month where max-update was found 
and compared max temperature for that particular day with reading of the reference website. When there was a considerable difference we calculated average and standard deviation for those differences. As, we have earlier stated that to determine validity, firstly we need to find those validity rules that are true (1) or false (0). They are represented as bool values. This process was performed for an entire year as well as for five separate years. Probability Validity was calculated for both scenarios so as to determine the more valid data set among the two data sets.

Table1 (a): representing update day, their corresponding actual and obtained max temperature values for data set 1 .

\begin{tabular}{|l|l|l|l|l|}
\hline Up_DATES & Actual & Obtained & Difference & bool \\
\hline $07-01-2012$ & 18 & 18 & 0 & 0 \\
\hline $09-02-2012$ & 23 & 23.6 & 0.6 & 1 \\
\hline $10-03-2012$ & 28 & 29.6 & 1.6 & 0 \\
\hline $15-04-2012$ & 34 & 34.8 & 0.8 & 1 \\
\hline $23-05-2012$ & 41 & 41.7 & 0.7 & 1 \\
\hline $19-06-2012$ & 31 & 31.8 & 0.8 & 1 \\
\hline $31-07-2012$ & 27 & 27.2 & 0.2 & 1 \\
\hline $14-08-2012$ & 25 & 25.4 & 0.4 & 1 \\
\hline $30-09-2012$ & 32 & 32.1 & 0.1 & 1 \\
\hline $27-10-2012$ & 28 & 28 & 0 & 0 \\
\hline $18-11-2012$ & 24 & 23.9 & 0.1 & 1 \\
\hline $22-12-2012$ & 22 & 21.8 & 0.2 & 1 \\
\hline
\end{tabular}

Table 1 (b): representing update day, their corresponding actual and obtained max temperature values for data set 2 .

\begin{tabular}{|l|l|l|l|l|}
\hline Up_DATES & Actual & Obtained & Difference & bool \\
\hline $18-01-2012$ & 24 & 26.8 & 2.8 & 1 \\
\hline $22-02-2012$ & 31 & 35.2 & 4.2 & 1 \\
\hline $12-03-2012$ & 31 & 32.78 & 1.78 & 1 \\
\hline $06-04-2012$ & 33 & 40.14 & 7.14 & 0 \\
\hline $31-05-2012$ & 39 & 47.76 & 8.76 & 0 \\
\hline $23-06-2012$ & 25 & 27.65 & 2.65 & 1 \\
\hline $25-07-2012$ & 29 & 28.23 & 0.77 & 0 \\
\hline $22-08-2012$ & 25 & 30.72 & 5.72 & 1 \\
\hline $15-09-2012$ & 28 & 26.72 & 1.28 & 0 \\
\hline $23-10-2012$ & 28 & 32.72 & 4.72 & 1 \\
\hline $12-11-2012$ & 25 & 30.78 & 5.78 & 1 \\
\hline $10-12-2012$ & 27 & 30.40 & 3.40 & 1 \\
\hline
\end{tabular}


Table 1 (c): Validity measure obtained from data set $1 \&$ data set 2.

\begin{tabular}{|l|l|l|}
\hline Measure & Data Set 1 & Data Set 2 \\
\hline Average & 0.46 & 4.08 \\
\hline Standard Deviation & 0.47 & 2.44 \\
\hline Upper limit & 0.93 & 6.52 \\
\hline Lower limit & 0.01 & 1.64 \\
\hline Probability Validity & $75 \%$ & $67 \%$ \\
\hline
\end{tabular}

Table 1(d): representing update day, their corresponding actual and obtained max temperature values for data set $1 \&$ data set 2 for five different years for February.

\begin{tabular}{|l|l|l|l|l|l|}
\hline Up_Dates & Actual & $\begin{array}{l}\text { Obtained for } \\
\text { Data Set 1 }\end{array}$ & $\begin{array}{l}\text { Obtained for } \\
\text { Data Set 2 }\end{array}$ & $\begin{array}{l}\text { Boolean value } \\
\text { for Data Set 1 }\end{array}$ & $\begin{array}{l}\text { Boolean value } \\
\text { for Data Set 2 }\end{array}$ \\
\hline $23-02-2010$ & 31 & 31.2 & 30.5 & 1 & 0 \\
\hline $27-02-2010$ & 27 & 30.2 & 33.5 & 0 & 0 \\
\hline $07-02-2011$ & 29 & 29.9 & 33.0 & 1 & 1 \\
\hline $14-02-2011$ & 31 & 31.2 & 35.7 & 1 & 1 \\
\hline $09-02-2012$ & 23 & 23.6 & 24.5 & 1 & 0 \\
\hline $22-02-2012$ & 31 & 31.4 & 35.2 & 1 & 1 \\
\hline $16-02-2013$ & 20 & 20.6 & 24.5 & 1 & 1 \\
\hline $07-02-2014$ & 28 & 29.5 & 31.8 & 1 & 0 \\
\hline $28-02-2014$ & 19 & 20.0 & 27.1 & 1 & 1 \\
\hline
\end{tabular}

Table 1(e): representing different measures obtained from data set $1 \&$ data set 2 when evaluated for five different years.

\begin{tabular}{|l|l|l|}
\hline Measure & Data Set 1 & Data Set 2 \\
\hline Average & 0.95 & 4.20 \\
\hline Standard Deviation & 0.88 & 2.16 \\
\hline Upper limit & 1.83 & 6.36 \\
\hline Lower limit & 0.07 & 2.04 \\
\hline Probability Validity & $89 \%$ & $56 \%$ \\
\hline
\end{tabular}

\section{Result 1:}

To determine probability validity, we used formula mentioned in (1). Formula was applied on the two datasets as shown in table 1(a) and table 1(b). Prob. Validity obtained for the two data sets 
was $75 \%$ and $67 \%$ as mentioned in table $1(\mathrm{c})$, which shows that data set 1 has higher chances of validity than data set 2 . Similarly, when Prob. Validity was evaluated for five different years, it was found that for data set 1 it came out to be $89 \%$ while in data set 2 it was $56 \%$, which further suggests that validity of data set 1 is more than data set 2 .

\subsection{Data Freshness:}

Timeliness of data processing has also been referred to as "perishable insights". If data is not collected and analyzed within the real time constraints, it no longer remains valid and is unusable. So, data freshness becomes one of the most important attributes for measuring the quality of data. It's a highly beneficial measure especially in case of an IoT application due to the heterogeneity of data sources, high interoperability and rapid generation of data from the sensors. Playing a major role for the analysis of data, it mainly refers to how often a new data is obtained.

As we have considered two separate data sets, so we obtained number of updates occurring in each month for both data sets shown in figure 3. When the number of updates is more, the guarantee for data freshness is established.

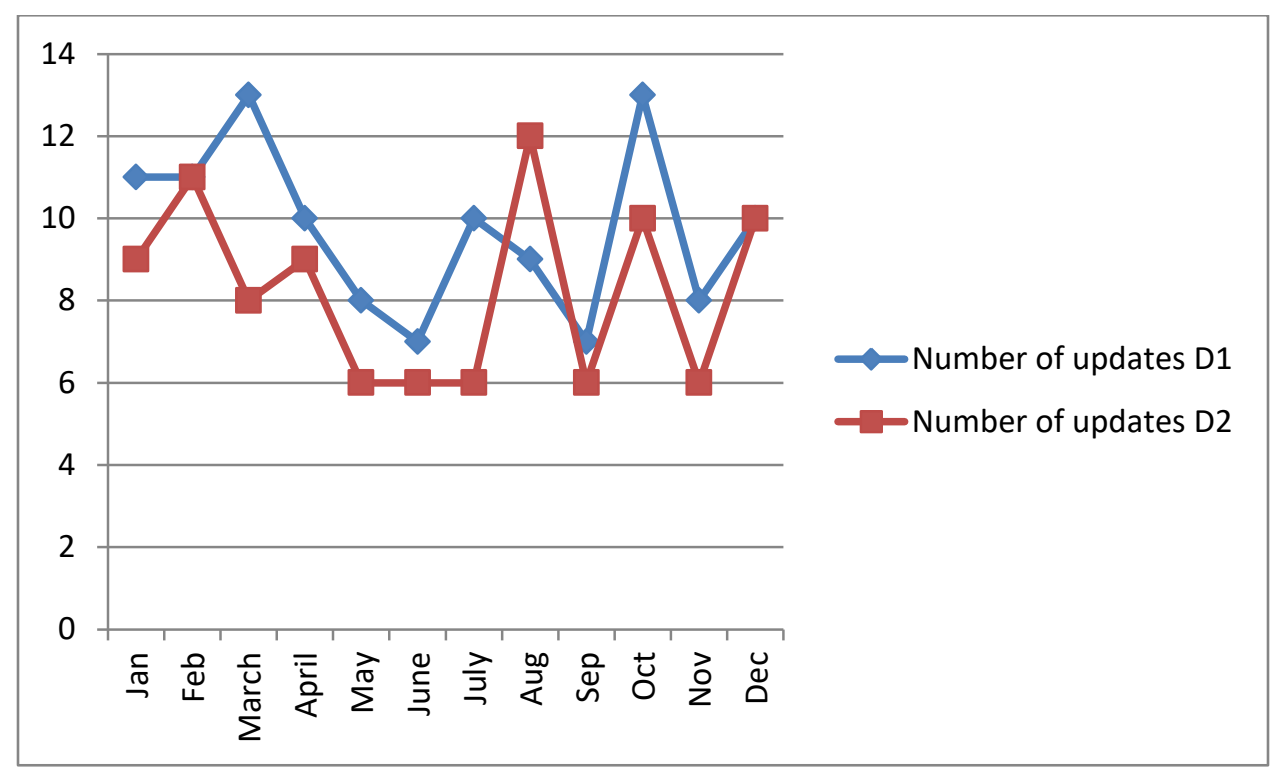

Figure 3: represents the number of updates occurring in each month for both data sets.

\section{Result 2:}

From the figure 3 we see that for the majority of months, number of updates is more for data set 1 as compared to data set 2 . Thus, we found data freshness more in data set 1 than data set 2 .

\subsection{Availability:}


Data availability refers to the process of conforming that data is available to the user when it is required. As we consider pervasive environment, we define availability with respect to an observation period and interval between updates.

$$
\text { Availability }=1-\frac{\max \left(0, \sum_{i=0}^{n}\left(t_{i+1}-t_{i}\right)-T^{e x p}\right)}{O P}
$$

Where $n=$ No. of updates

OP=observation period (30days)

$t_{i}=$ time interval between successive updates.

Table 2 (a): Availability for Data set 1.

\begin{tabular}{|l|l|l|}
\hline Month & $T^{\text {exp }}=10$ days & $T^{\text {exp }}=15$ days \\
\hline Jan & 0.50 & 0.67 \\
\hline Feb & 0.40 & 0.57 \\
\hline March & 0.37 & 0.53 \\
\hline April & 0.47 & 0.63 \\
\hline May & 0.47 & 0.63 \\
\hline June & 0.53 & 0.70 \\
\hline July & 0.30 & 0.47 \\
\hline Aug & 0.33 & 0.50 \\
\hline Sep & 0.33 & 0.50 \\
\hline Oct & 0.30 & 0.47 \\
\hline Nov & 0.63 & 0.80 \\
\hline Dec & 0.40 & 0.57 \\
\hline
\end{tabular}

Table 2 (b): Availability for Data set 2.

\begin{tabular}{|l|l|l|}
\hline Month & \multicolumn{1}{|c|}{$T^{\exp =10}$} & \multicolumn{1}{c|}{$T^{\text {exp }}=15$} \\
\hline Jan & 0.53 & 0.70 \\
\hline Feb & 0.40 & 0.57 \\
\hline March & 0.40 & 0.57 \\
\hline April & 0.53 & 0.70 \\
\hline May & 0.30 & 0.47 \\
\hline June & 0.50 & 0.67 \\
\hline July & 0.33 & 0.50 \\
\hline Aug & 0.30 & 0.47 \\
\hline Sep & 0.33 & 0.50 \\
\hline Oct & 0.30 & 0.47 \\
\hline Nov & 0.93 & 1.00 \\
\hline Dec & 0.43 & 0.60 \\
\hline
\end{tabular}


We can view the performance of availability metric of two data sets for two different expiration times through figure 4 (a) and (b).

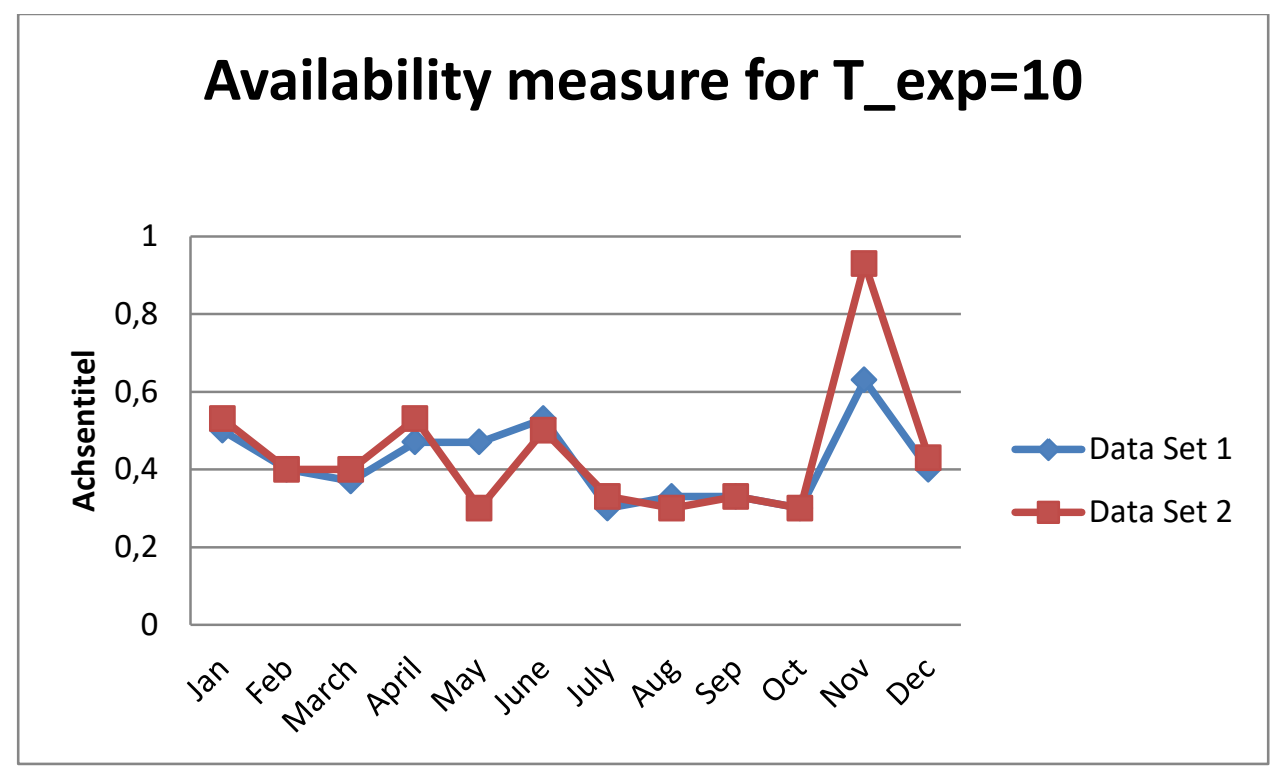

(a)

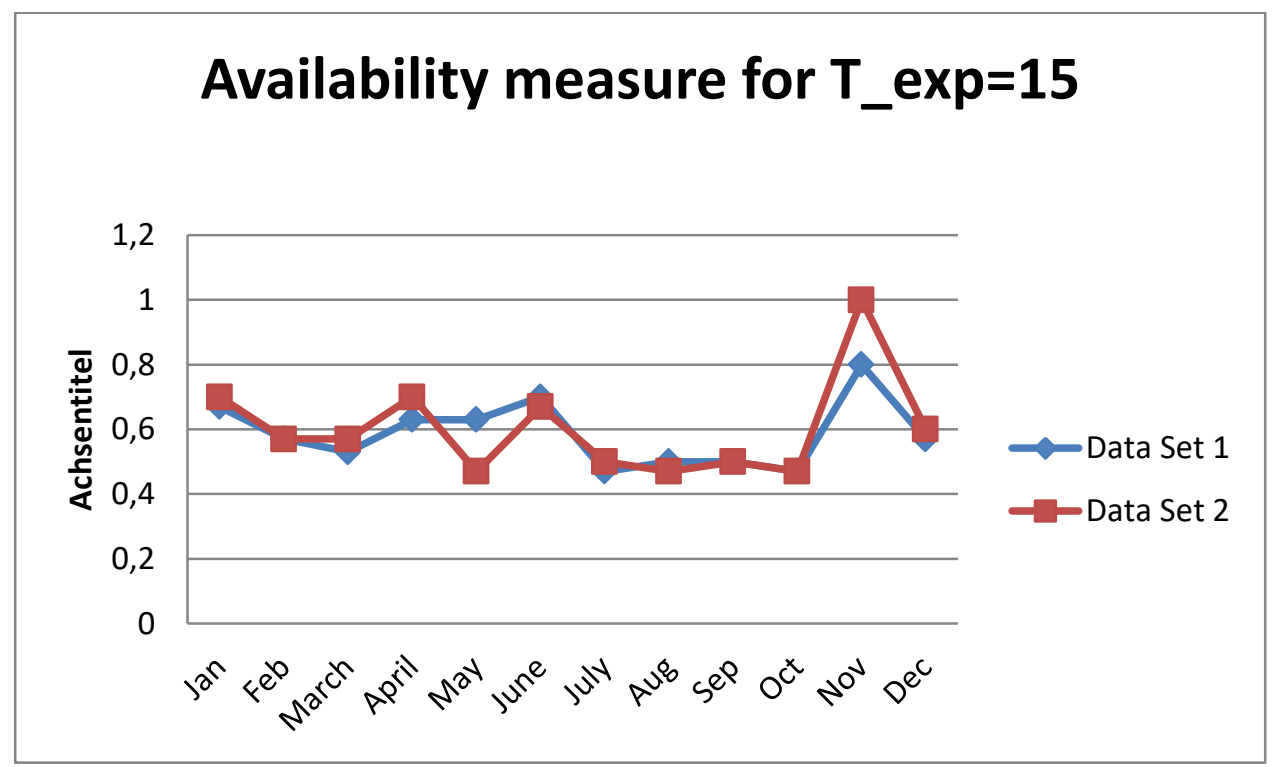

(b)

Figure 4: Availability metric obtained (a) expiration time:10 days (b) expiration time:15 days.

\section{Result 3:}

In order to determine availability, (2) was used. With the help of it, availability was calculated for each month for two different expiry time for both data sets as shown in table 2(a) and 2(b). 
When we observe the availability metric obtained from figure 4(a) and figure 4(b), it was found that data set 2 was better in terms of availability as compared to data set 1 .

\subsection{Completeness}

In case of contextual and representational quality of data [21], concise representation, completeness, Value added and relevancy are most important. Completeness generally refers to the property of any system that doesn't suffer from data loss. It defines that whether the data source is capable of providing all the information which it mentioned and is required by the application [20]. Completeness can be evaluated at different levels such as:

- Scheme Completeness (entities and attributes are missing)

- Column Completeness (column values are missing)

- Population Completeness (column doesn't contains all the samples of population)

Mathematically, it can be defined as:

$$
\text { Completeness }=1-\frac{\text { no.of incomplete items }}{\text { total no.of items }}
$$

In our study, completeness is directly proportional to the number of updates. Since, more the number of updates less will be the chances of missing the updated data. As, we are computing completeness annually, it will be depending upon the update values of individual month and will be calculated in a relative term with respect to the maximum number of updates found in a yearly dataset i.e.

$$
\text { Completeness }=\frac{\sum u_{i}}{\sum u_{\max }}
$$

Where: $\quad u_{i}=$ no. of updates occurring in a month

$$
u_{\max }=\text { Maximum no. of updates occuring in a month. }
$$

Table 3: Representing number of updates obtained for each month for the two datasets.

\begin{tabular}{|l|l|l|}
\hline Month & Updates in Dataset 1 & Updates in Dataset2 \\
\hline Jan & 11 & 9 \\
\hline Feb & 11 & 11 \\
\hline March & 13 & 8 \\
\hline April & 10 & 9 \\
\hline May & 8 & 6 \\
\hline June & 7 & 6 \\
\hline July & 10 & 6 \\
\hline Aug & 9 & 12 \\
\hline Sep & 7 & 6 \\
\hline Oct & 13 & 10 \\
\hline
\end{tabular}




\begin{tabular}{|l|l|l|}
\hline Nov & 8 & 6 \\
\hline Dec & 10 & 10 \\
\hline
\end{tabular}

\section{Result 4:}

For evaluating completeness for the data sets, formula mentioned in (4) was used. Number of updates obtained for each month can be observed in table 3. Using these updates value, it was substituted in (4). On evaluating completeness, it was found that data set 1 had relative completeness measure of $75 \%$ while data set 2 had $69 \%$. Thus, it was concluded that data set 1 is more complete than data set 2 .

\subsection{Usability}

Data may be incomplete by design or due to operational requirements. Moreover, when data quality is measured on the basis of context, the same data is needed for multiple tasks but different required characteristics. As per application requirements, the same data representation may render useless if it requires aggregates or fields of data that do not exist. This causes poor relevancy to the application and is considered incomplete for analysis, thereby reducing its usability. Usability has also been correlated with the interpretability and ease of understanding of a data that may require expert opinion due to the enormous volume or simply the lack of common interpretability. We measure the usability of data by measuring the deviation from their aggregates to the individual values. The degree of aggregation and variation in their representations have been computed and as their maximum tolerable anomaly which is represented in table 4 .

Table 4: Represents Usability metrics obtained for both data sets for February.

\begin{tabular}{|c|c|c|}
\hline Criteria & Data Set 1 & Data Set 2 \\
\hline upper_L= & 0.892681 & 5.982644 \\
\hline lower_L= & 0.169388 & 1.573908 \\
\hline Usability $=$ & $69 \%$ & $86 \%$ \\
\hline
\end{tabular}

\section{Result 5:}

To decide usability, randomly a month was selected (February in our case). All the entries of the month were compared with the entries in [21] for the same month. Set of valid entries were determined by the interval limits mentioned in table 4 . Usability rate of $69 \%$ and $86 \%$ was found for the data sets respectively, which shows data set 2 had better usability than data set 1 .

\section{Conclusion:}


Metrics that have been defined for measuring the data quality for an IoT application have been implemented on weather data to display its significance. In earlier research works, metrics for data quality had not been applied for aggregated data. Thus, we used five metrics namely validity, data freshness, availability, completeness and usability for observing the data quality on a collective basis. The result shows that validity, freshness and availability metrics for data set 1 is more than data set 2 . We also depicted that completeness and usability of data collected may vary but is always aligned with the validity of data. A more valid data will be more complete, more usable, more available and also up-to-date. In future we aim to implement other context based metrics on data obtained from IoT based applications.

\section{Declarations:}

- Funding: Not Applicable

- Conflicts of Interests/ Competing Interests: Not Applicable

- Availability of Data \& Material: The data sets which were used for the study have been mentioned in the reference section. Please refer ref. number [21, 22 and 23]. [21, 22] are the two data sets that have been used for the study while [23] acts as a reference point.

- Code Availability: Code can be provided if the editor requires.

\section{References:}

1. Chen, Hao, XueqinJia, and Heng Li. "A brief introduction to IoT gateway." IET international conference on communication technology and application (ICCTA 2011). IET, 2011.

2. Alam, Mahbubul, Rasmus H. Nielsen, and Neeli R. Prasad. "The evolution of M2M into IoT." 2013 First International Black Sea Conference on Communications and Networking (BlackSeaCom). IEEE, 2013.

3. Udoh, Itorobong S., and Gerald Kotonya. "Developing IoT applications: challenges and frameworks." IET Cyber-Physical Systems: Theory \& Applications 3.2 (2018): 65-72.

4. Babovic, Zoran B., JelicaProtic, and VeljkoMilutinovic. "Web performance evaluation for internet of things applications." IEEE Access 4 (2016): 6974-6992.

5. Fenton, Norman E., and Martin Neil. "Software metrics: roadmap." Proceedings of the Conference on the Future of Software Engineering. 2000. 
6. Miguel, José P., David Mauricio, and Glen Rodríguez. "A review of software quality models for the evaluation of software products." arXiv preprint arXiv:1412.2977 (2014).

7. Bhatti, Shahid Nazir. "Why quality? iso 9126 software quality metrics (functionality) support by uml suite." ACM SIGSOFT Software Engineering Notes 30.2 (2005): 1-5.

8. Power, Alexander, and Gerald Kotonya. "Providing fault tolerance via complex event processing and machine learning for iot systems." Proceedings of the 9th International Conference on the Internet of Things. 2019.

9. Nieh, Jason, and Monica S. Lam. "A SMART scheduler for multimedia applications." ACM Transactions on Computer Systems (TOCS) 21.2 (2003): 117-163.

10. Kuemper, Daniel, et al. "Valid. IoT: a framework for sensor data quality analysis and interpolation." Proceedings of the 9th ACM Multimedia Systems Conference. 2018.

11. Jayashree, L. S., S. Arumugam, and A. R. Meenakshi. "A communication-efficient framework for outlier-free data reporting in data-gathering sensor networks." International Journal of Network Management 18.5 (2008): 437-445.

12. Nisa, Ishrat Un, and Syed Nadeem Ahsan. "Fault prediction model for software using soft computing techniques." 2015 International Conference on Open Source Systems \& Technologies (ICOSST). IEEE, 2015.

13. Ravichandran, Jaichandran, and Anthony Irudhayaraj Arulappan. "Data validation algorithm for wireless sensor networks." International Journal of Distributed Sensor Networks 9.12 (2013): 634278.

14. Klima, Matej, et al. "Quality and reliability metrics for IoT systems: a consolidated view." International Summit Smart City $360^{\circ}$. Springer, Cham, 2020.

15. Karkouch, Aimad, et al. "Data quality in internet of things: A state-of-the-art survey." Journal of Network and Computer Applications 73 (2016): 57-81.

16. Pipino, Leo L., Yang W. Lee, and Richard Y. Wang. "Data quality assessment." Communications of the ACM 45.4 (2002): 211-218.

17. Byabazaire, John, Gregory O'Hare, and Declan Delaney. "Data Quality and Trust: Review of Challenges and Opportunities for Data Sharing in IoT." Electronics 9.12 (2020): 2083.

18. Kaiser, Marcus, Mathias Klier, and Bernd Heinrich. "How to measure data quality?-a metric-based approach." ICIS 2007 Proceedings (2007): 108. 
19. Buchholz, Thomas, Axel Küpper, and Michael Schiffers. "Quality of context: What it is and why we need it." Workshop of the HP OpenView University Association. 2003.

20. Thomas, Michael Onuoha, and Babak Bashari Rad. "Reliability evaluation metrics for internet of things, car tracking system: a review." Int. J. Inf. Technol. Comput. Sci.(IJITCS) 9.2 (2017): 1-10.

21. https://mausam.imd.gov.in/

22. http://amsskolkata.gov.in/mc/ranchi

23. https://www.timeanddate.com, last accessed: July 2020. 